\title{
Recent pioneers
}

\author{
Lawrence W. Jones $^{\text {a }}$ \\ University of Michigan, Ann Arbor, Michigan, USA
}

Abstract. Recognition of outstanding Russian cosmic ray physicists and earlier meetings of this Symposium.

\section{Introduction}

I have had the pleasure of attending almost all of these International Symposia on Very High Energy Cosmic Ray Interactions since their origin in 1980. We had the privilege of hosting the 1992 meeting at my home institution, the University of Michigan in Ann Arbor, Michigan, U.S.A. The meetings were always informative, pleasant, and lively, with a diverse international attendance of physicists from around the world. To be sure, there was certainly some overlap in topics discussed and those in attendance at the International Cosmic Ray Conferences held during the summer on alternate years. However, our ISVHECRI meetings have always focused more narrowly on particle interactions and air showers, and the participation at the meetings is smaller and more intimate.

\section{Pioneers}

The community of physicists at the meetings was always pleasant and stimulating. Essentially everyone had been involved in a significant program, usually a cosmic ray experiment. In the last century (before the year 2000), almost all of the significant experiments were on the ground, including experimental sites at mountain elevations. To be sure, there were also balloon experiments, which were of limited weight and area.

At and near sea level, the most common experiments were (and still are) air shower arrays, where the data collect particles (electrons, muons, and gamma rays, mostly) from interactions in the atmosphere above. However at high mountain elevations, primary interactions are less uncommon, and the interactions of cosmic ray protons can be (and, in the last century, were) studied.

Two experimental programs of interest in those days were the studies in Bolivia on Mt. Chacaltaya (at an elevation of 5200 meters) and in the Russian Pamir mountains (at 4370 meters). This Russian experiment was named "Pamir".
The Russian physicist who directed the Pamir program was Serguei Slavatinsky, whose home base was the Lebedev Physical Institute in Moscow. Without going into details on the physics results from Pamir, it is appropriate to note that it was a very active and productive program in the 1980s. I must also note that Slavatinsky was a most pleasant and delightful friend; he was an active participant in these ISVHECRI Symposia, as well as at the ICRC meetings. Sadly, he died several years ago, so is no longer with us. However, in view of his significant leadership of the Pamir program with his reporting and communication, he certainly merits recognition as one of the Russian "Pioneers".

I will add, as an appropriate citation to his work and communication to the cosmic ray community, reference to the paper he presented at our 1992 Michigan meeting of the ISVHECRI. His was the first paper presented there; entitled "Ordinary and Unordinary Results of Experiment "Pamir" on Interactions of Hadrons with Energies at 10-100 PeV" [1].

Of course there are many other significant international physicists, who merit recognition. For example, Sir Arnold Wolfendale from England was an earlier regular attendee at these meetings, and always had relevant and interesting contributions. Gaurang Yodh, from the U.S. but of Indian origin, has also been a lively contributer; he is currently directing HAWC, a very productive mountain Cherenkov air-shower array in Mexico. Oscar Saavedra, from Bolivia (and now in Italy) has championed the Mt. Chacaltaya program. Nor should we forget the valuable contributions made over the years by A.E. Chudakov, G. Zatsepin, S.I. Nikolsky, J. Linsley all sadly deceased.

\section{References}

[1] S.A. Slavatinsky, pp. 3-27 AIP Conference Proceedings 276; Very High Energy Cosmic Ray Interactions VIIth International Symposium (1992)

\footnotetext{
a e-mail: 1wjones@umich.edu

(C) The Authors, published by EDP Sciences. This is an Open Access article distributed under the terms of the Creative Commons Attribution License 4.0 (http://creativecommons.org/licenses/by/4.0/).
} 